\title{
Protective effects of wogonin on lipopolysaccharide-induced inflammation and apoptosis of lung epithelial cells and its possible mechanisms
}

\author{
Jinlin $\mathrm{Ge}^{1}$, Huanhuan Yang ${ }^{1}$, Yufeng Zeng ${ }^{1}$ and Yunjie Liu ${ }^{2 *}$
}

\author{
${ }^{*}$ Correspondence: \\ liuyunjie18@126.com \\ 2 Department of Respiratory \\ and Critical Care Medicine, \\ The Second People's Hospital \\ of Nantong, 298 Xinhua \\ Road, Chongchuan District, \\ Nantong 226002, Jiangsu, \\ China \\ Full list of author information \\ is available at the end of the \\ article
}

\begin{abstract}
Background: Wogonin (5, 7-dihydroxy-8-methoxyflavone) is a natural di-hydroxyl flavonoid extracted from the root of Scutellaria baicalensis Georgi. This paper was intended to investigate the mechanism of action of wogonin in alleviating the inflammation and apoptosis in acute lung injury (ALI).

Materials and methods: Lipopolysaccharide (LPS) was used to establish the in vitro model of ALI. After wogonin treatment, the cell viability and apoptosis of LPS-induced A549 cells were, respectively, measured by CCK-8, TUNEL assays and acridine orange/ ethidium bromide dual staining, while the contents of inflammatory cytokines and oxidative stress markers were estimated by RT-GPCR, ELISA assay, western blot analysis and commercial kits. Western blot was also conducted to assess the expression of proteins involved. Subsequently, the effect of wogonin on the sirtuin 1 (SIRT1)-mediated highmobility group box 1 protein (HMGB1) deacetylation was investigated. SIRT1 inhibitor EX527 was used to evaluate the regulatory effects of wogonin on SIRT1-mediated HMGB1 deacetylation in A549 cells under LPS stimulation.
\end{abstract}

Results: LPS induced inflammation, oxidative stress and apoptosis of A549 cells, which was abolished by wogonin. It was also found that wogonin promoted the HMGB1 deacetylation, accompanied by upregulated SIRT1 expression. However, SIRT1 inhibitor EX527 partially reversed the protective effects of wogonin on the inflammation and apoptosis of LPS-induced A549 cells.

Conclusion: Wogonin alleviated the inflammation and apoptosis in LPS-induced A549 cells by SIRT1-mediated HMGB1 deacetylation, which might represent the identification of a novel mechanism by which wogonin exerts protective effects on ALI and provide ideas for the application of wogonin to ALI treatment.

Keywords: Acute lung injury, Wogonin, Inflammation, SIRT1, HMGB1 deacetylation author(s) and the source, provide a link to the Creative Commons licence, and indicate if changes were made. The images or other third party material in this article are included in the article's Creative Commons licence, unless indicated otherwise in a credit line to the material. If material is not included in the article's Creative Commons licence and your intended use is not permitted by statutory regulation or exceeds the permitted use, you will need to obtain permission directly from the copyright holder. To view a copy of this licence, visit http:// creativecommons.org/licenses/by/4.0/. The Creative Commons Public Domain Dedication waiver (http://creativecommons.org/publicdomain/zero/1.0/) applies to the data made available in this article, unless otherwise stated in a credit line to the data. 


\section{Background}

Acute lung injury (ALI) is clinically documented as the result of the pathological states including sepsis, pneumonia, trauma, and acute pancreatitis [1]. It is often seen in patients who are admitted into the intensive care units and shows a high mortality rate [2]. Those who survive from ALI are usually confronted with inferior quality of life [3]. Although achievements have been made in understanding the pathophysiology of ALI, the treatment efficacy of current therapies remains limited, which cannot fulfill the expectations of the patients and their families [4]. Thus, it is of great urgency to identify new therapies or targets for the treatment of ALI.

Wogonin (5,7-dihydroxy-8-methoxyflavone), the structure of which is shown in Fig. 1A, is a natural di-hydroxyl flavonoid extracted from the root of Scutellaria baicalensis Georgi [5]. Its significant anti-inflammatory properties and its application in the treatment of inflammatory diseases have been widely reported by a large number of studies. For instance, wogonin potentially improved the lung edema in the murine and protected them from lipopolysaccharide (LPS)-induced ALI by blocking p38 mitogen activated protein kinase (MAPK) and c-Jun NH(2)-terminal kinase (JNK) phosphorylation [6]. Wogonin also reduced LPS-induced neutrophil infiltration, production of proinflammatory cytokines, expression of adhesion molecules and suppressed LPS-induced ALI in mice [7]. Current evidence suggested that the inflammatory response and ALI induced by LPS could be attenuated as a result of wogonin treatment by peroxisome proliferator-activated receptor gamma (PPAR $\gamma$ )-mediated

A<smiles>COc1c(O)cc(O)c2c(=O)cc(-c3ccccc3)oc12</smiles>

C

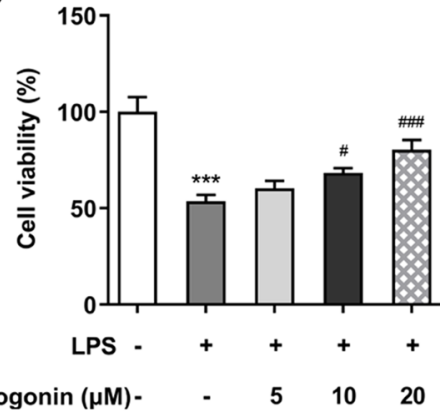

B

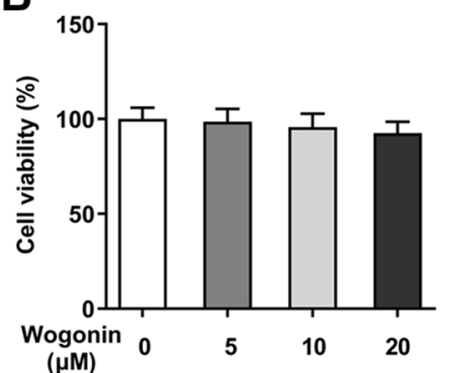

D

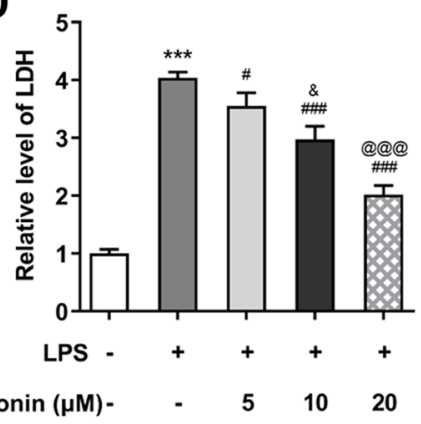

Fig. 1 Effect of wogonin on the viability of LPS-induced A549 cells. A The chemical structure of wogonin. B The viability of A549 cells exposed to wogonin was determined by a CCK-8 assay. C The viability of LPS-induced A549 cells exposed to wogonin was estimated by a CCK-8 assay. D The LDH activity in LPS-induced A549 cells exposed to wogonin was determined by a LDH assay kit. Data were obtained from three independent experiments $(n=3)$. ${ }^{* *} P<0.001$ vs. control; ${ }^{\#} P<0.05,{ }^{\# \#} P<0.001$ vs. LPS; ${ }^{\circledR} P<0.05$ vs. LPS + $5 \mu$ M wogonin; $@ @ ~ P<0.001$ vs. LPS $+10 \mu \mathrm{M}$ wogonin 
NF-кB pathway [8]. Emerging study showed that wogonin reduced cecal ligation and puncture (CLP)-induced high-mobility group box 1 protein (HMGB1) production and HMGB1dependent inflammation, and lowered sepsis-related morbidity and the risk of lung injury [9]. In the LPS-induced in vitro and in vivo ALI model, another report demonstrated that the inhibition of HMGB1 and other inflammatory mediators via ulinastatin evidently alleviated the manifestations of ALI [10]. And previous studies confirmed that LPS-induced human lung epithelial cells (A549) has been widely used as an in vitro model for ALI [11-13].

Sirtuin 1 (SIRT1), the downstream effector of adenosine monophosphate activated protein kinase (AMPK), has been suggested to play significant roles in the regulation of adiponectin release [14]. Wogonin supplementation remarkably increased the AMPK phosphorylation and SIRT1 expression, whereas inhibition of AMPK or SIRT1 reduced the effects of wogonin on the production or release of adiponectin [14]. It was previously reported that SIRT1-modulated HMGB1 deacetylation inhibited acute kidney injury incurred by sepsis [15].

In the present study, we aimed to investigate the action mechanism of wogonin in alleviating the inflammation and apoptosis of LPS-induced human lung epithelial cells, which might provide a more comprehensive insight for the treatment of ALI by wogonin.

\section{Results}

\section{Effects of wogonin on the viability and apoptosis of LPS-induced A549 cells}

To observe the effect of wogonin on ALI, we first speculated if the viability of normal A549 cells could be damaged by wogonin. As exhibited in Fig. 1B, wogonin did no harm to A549 cells without receiving any treatment, suggesting the safety of wogonin treatment in A549 cells at the concentrations of $0,5,10$, and $20 \mu \mathrm{M}$. After LPS stimulation for $24 \mathrm{~h}$, the viability of A549 cells was markedly damaged, which was counteracted by wogonin in a concentration-dependent manner (Fig. 1C). Furthermore, results from LDH assay showed that significant increase in LDH activity in LPS-treated A549 cells was reduced after increasing doses of wogonin were administrated (Fig. 1D). Next, we tested the effect of wogonin on the apoptosis of LPS-induced A549 cells. As exhibited in Fig. 2A, TUNEL staining demonstrated that LPS exposure dramatically enhanced the apoptosis of A549 cells compared with the control group, which was reduced by wogonin in a dose-dependent manner. Besides, apparent downregulation in Bcl-2 expression and upregulation in Bax, Cyto-C and cleaved caspase 3 expression were observed in the LPS-exposed group when compared to the control group, which was restored by wogonin treatment (Fig. 2B). Consistently, results of AO/EB double fluorescence assays presented in Fig. 3 suggested that LPS led to the elevated cell apoptosis rate as comparison to the control group, while the EB-positive cells were notably reduced after wogonin addition when compared to the LPS group. Thus, wogonin restores the viability and reduces the apoptosis of A549 cells induced by LPS.

\section{Effects of wogonin on the inflammation and oxidative stress of LPS-induced A549 cells}

To explore the anti-inflammatory and anti-oxidant abilities of wogonin in ALI, the inflammation and oxidative stress of LPS-induced A549 cells treated with wogonin were, respectively, detected. As seen in Fig. 4A-C, the mRNA levels of inflammatory cytokines (TNF- $\alpha$, IL-6 and IL-1 $\beta$ ) measured by RT-qPCR rose to notably high levels upon LPS stimulation, whereas wogonin treatment led to the reduction in their expression. Consistently, the results of ELISA indicated that LPS induction led to the significant increase in TNF- $\alpha$, IL- 6 and IL- $1 \beta$ contents 

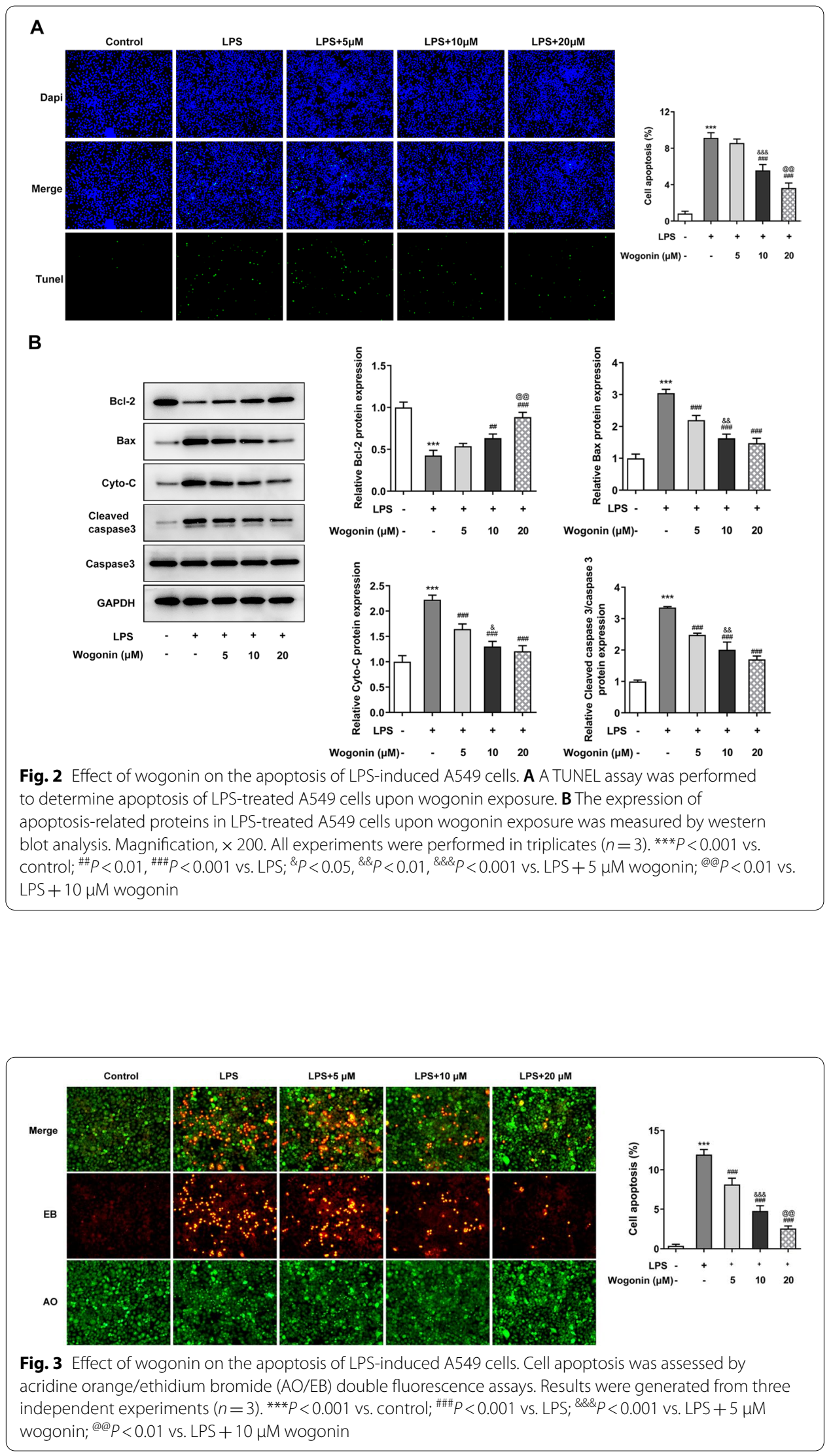
compared with the untreated group, which were dose-dependently decreased after wogonin treatment (Fig. 4D-F). Importantly, cyclooxygenase-2 (Cox-2) and phospho-nuclear factor (NF)-кB p65 (p-NF-кB p65), which are inflammation-related markers, were down-regulated by LPS while upregulated by wogonin (Fig. 4G). In addition, LPS resulted in decreased activities of SOD and GSH-Px and increased contents of MDA and ROS, which was reversed by wogonin (Fig. $4 \mathrm{H}-\mathrm{K})$. Taken together, wogonin alleviates the inflammation and oxidative stress of LPS-induced A549 cells.

\section{Regulation of wogonin in SIRT1-mediated HMGB1 deacetylation}

To confirm our speculation that wogonin exerted protective effects on LPS-induced A549 cell injury by SIRT1-mediated HMGB1 deacetylation, we carried out western blot to detect the protein levels of involved factors. Evidently, LPS suppressed the expression of SIRT1

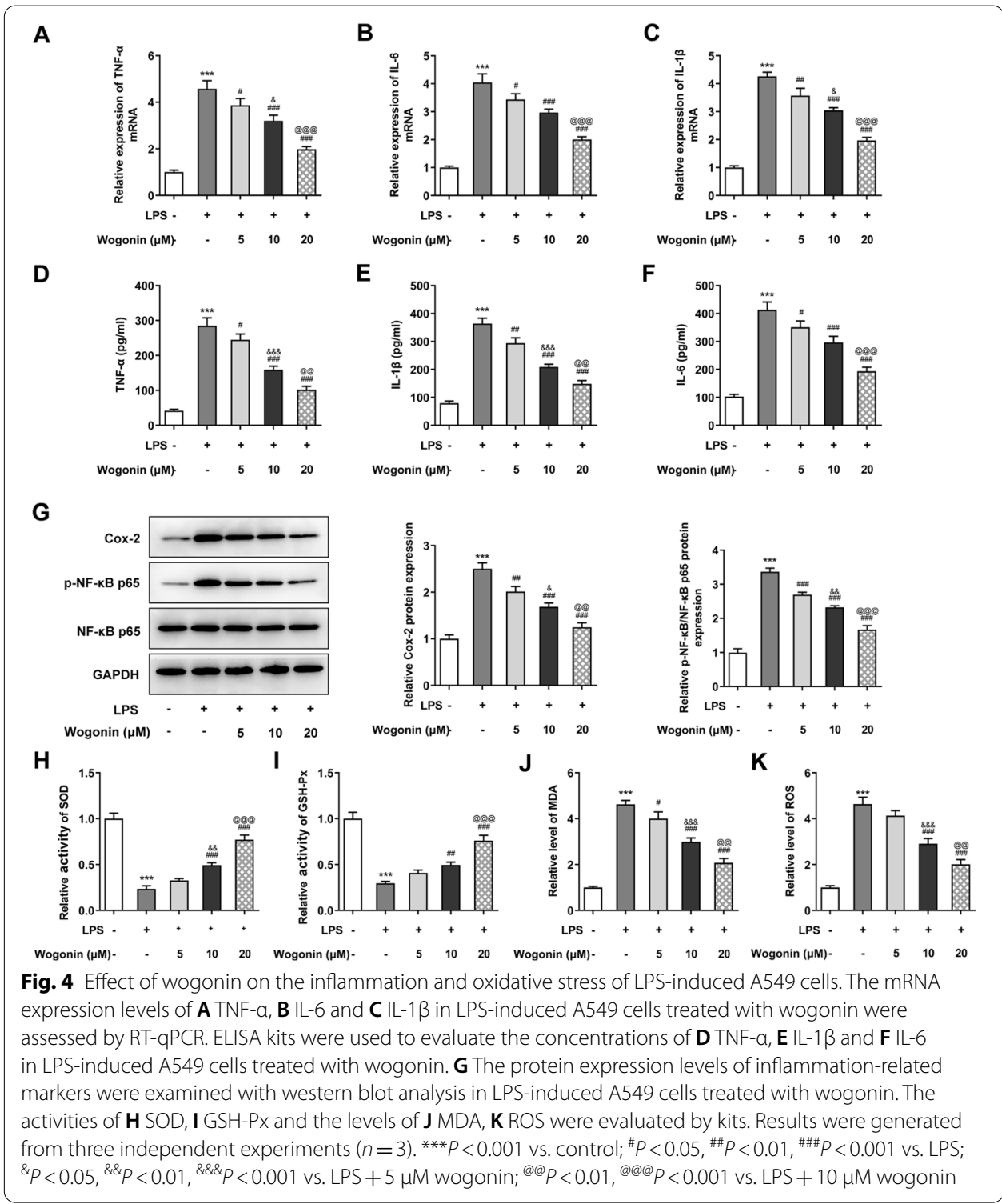


and promoted the translocation of HMGB1 from the nucleus to the cytoplasm, along with HMGB1 acetylation. However, this trend was reversed by wogonin exposure to LPS-induced A549 cells (Fig. 5). Thus, wogonin treatment regulates SIRT1-mediated HMGB1 deacetylation in LPS-induced A549 cells.

\section{The regulation of SIRT1 inhibitor EX527 on the cellular behaviors of LPS-induced A549 cells treated with wogonin}

To verify the role of SIRT1 in the underlying mechanism by which wogonin impacted the inflammation and apoptosis of LPS-induced A549 cells, we used EX527, an SIRT1 inhibitor, to treat LPS-induced A549 cells for $24 \mathrm{~h}$. As shown in Fig. 6A, the addition of EX527 lessened SIRT1 expression, impeded the nucleo-cytoplasmic transport of HMGB1, and stimulated HMGB1 deacetylation, as compared to LPS + Wogonin group. Further CCK-8 and LDH assays displayed that EX527 damaged the restorative effect of wogonin on the viability of LPSinduced A549 cells (Fig. 6B). Besides, the effects of wogonin on the expression of Bcl-2, Bax, Cyto-C and cleaved caspase 3 expression were partly alleviated by EX527 in LPS-induced A549 cells (Fig. 6C). Finally, the variation trends of the inflammatory factors (TNF- $\alpha$, IL- 6 and IL-1 $\beta$ ), inflammation-related proteins (Cox-2 and p-NF-kB p65) and oxidative stress markers (SOD, GSH-Px and MDA) in LPS-induced A549 cells co-treated with wogonin and EX527 demonstrated that EX527 damaged the inhibitory effects of wogonin on the inflammation and oxidative stress in LPS-induced A549 cells (Fig. 7A-K). Therefore, SIRT1 inhibitor EX527 reverses the protective effects of wogonin on the malignant phenotypes of LPS-induced A549 cells.

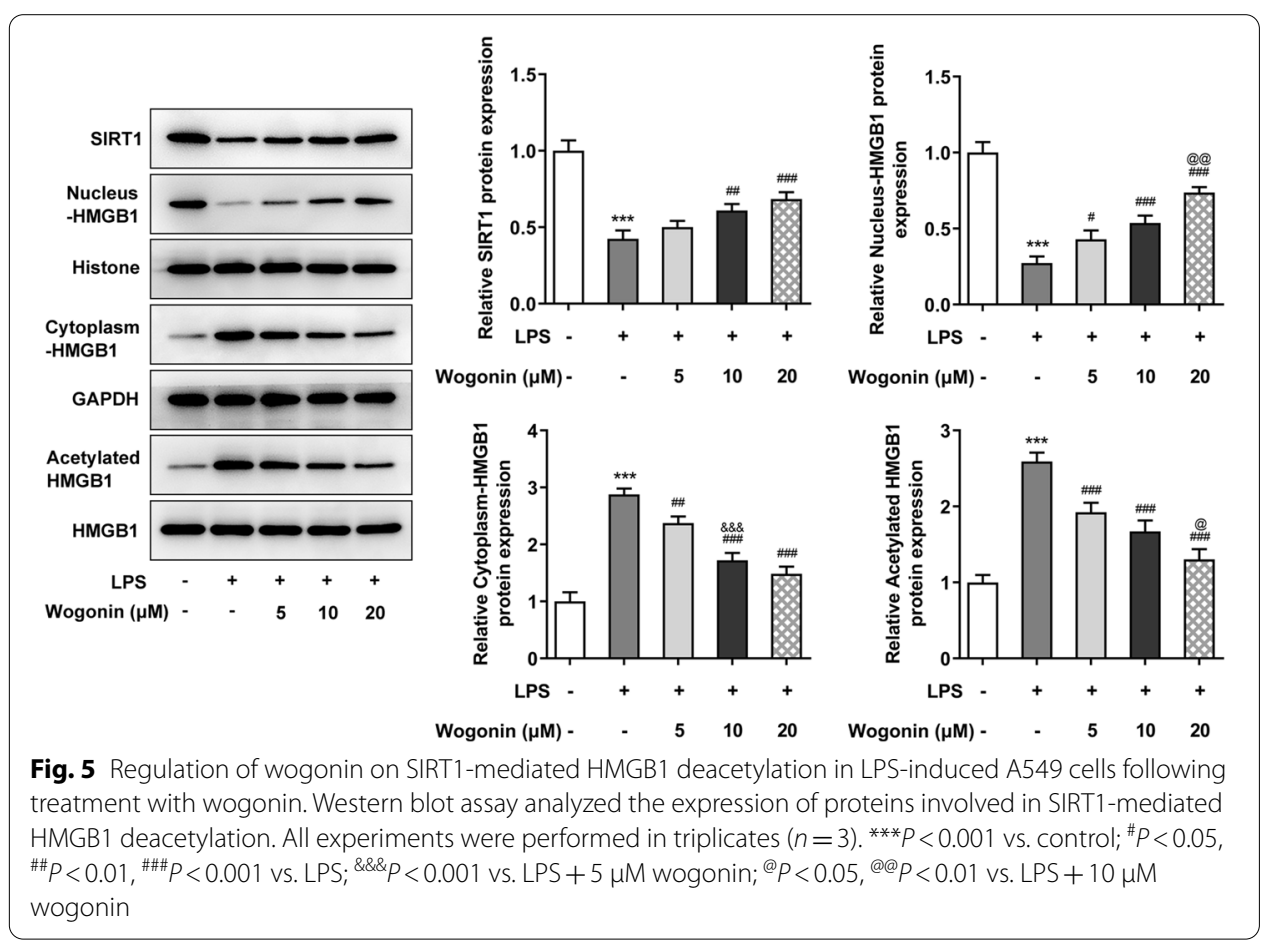




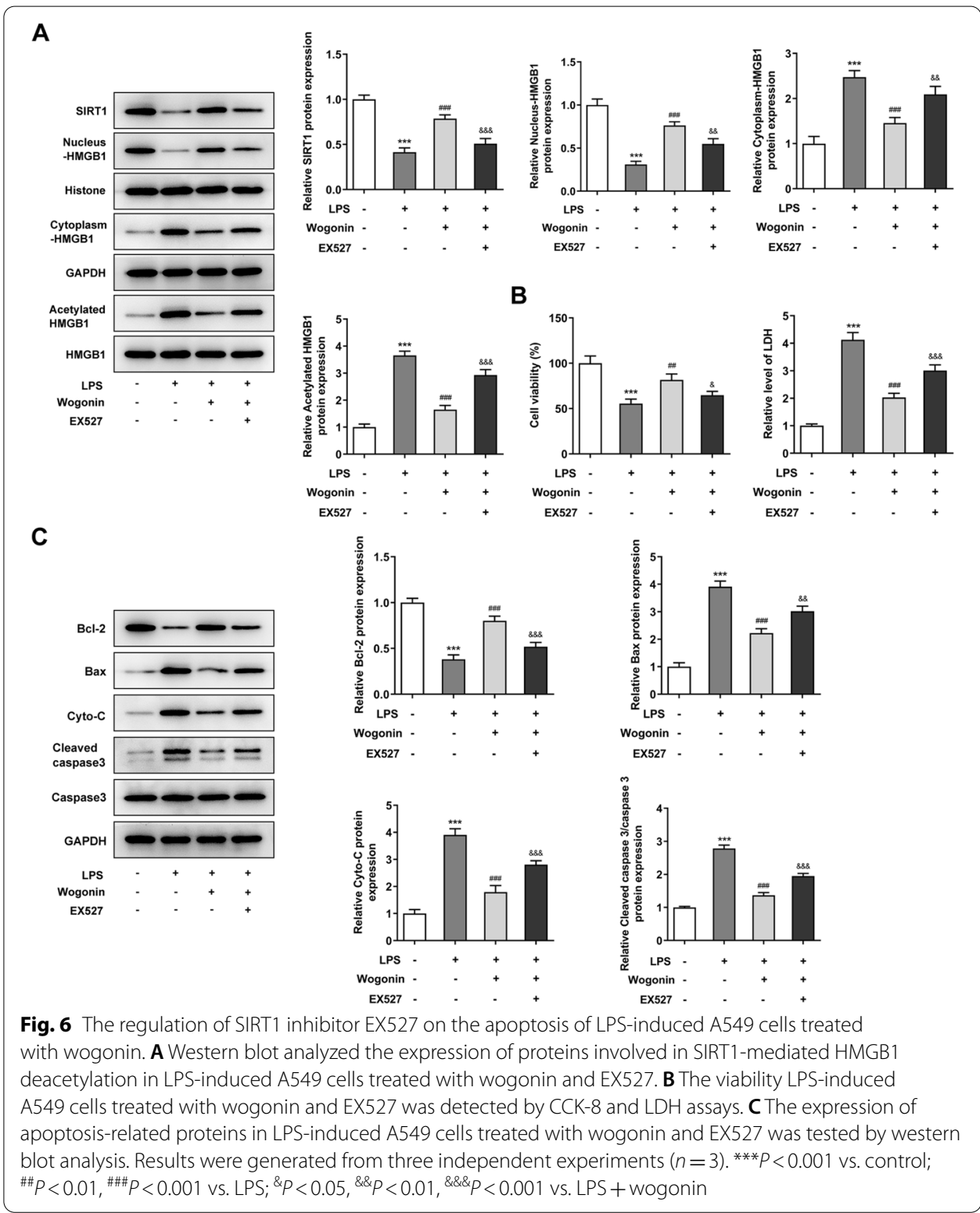

\section{Discussion}

Currently, many experts have recognized wogonin as potent agents for the treatment of inflammatory diseases. Its efficacy in alleviating inflammatory responses was reportedly attributed to the suppression of NF-KB and activation of nuclear factor erythroid 2-related factor 2 (Nrf2) signaling pathways [16]. Interestingly, previous reports associated the anti-inflammatory effect with the mediation of iNOS and Cox-2 expression or the activation of reactive oxygen species (ROS)/ERK/Nrf2 signaling pathways $[5,17]$. Consistent with previous findings displaying no effect of wogonin on chondrocyte viability, we found virtually no significant cytotoxic effect of wogonin on normal A549 cells, supporting our further exploration into the underlying mechanism by which wogonin impacted in vitro ALI cell model [17]. Oxidative 


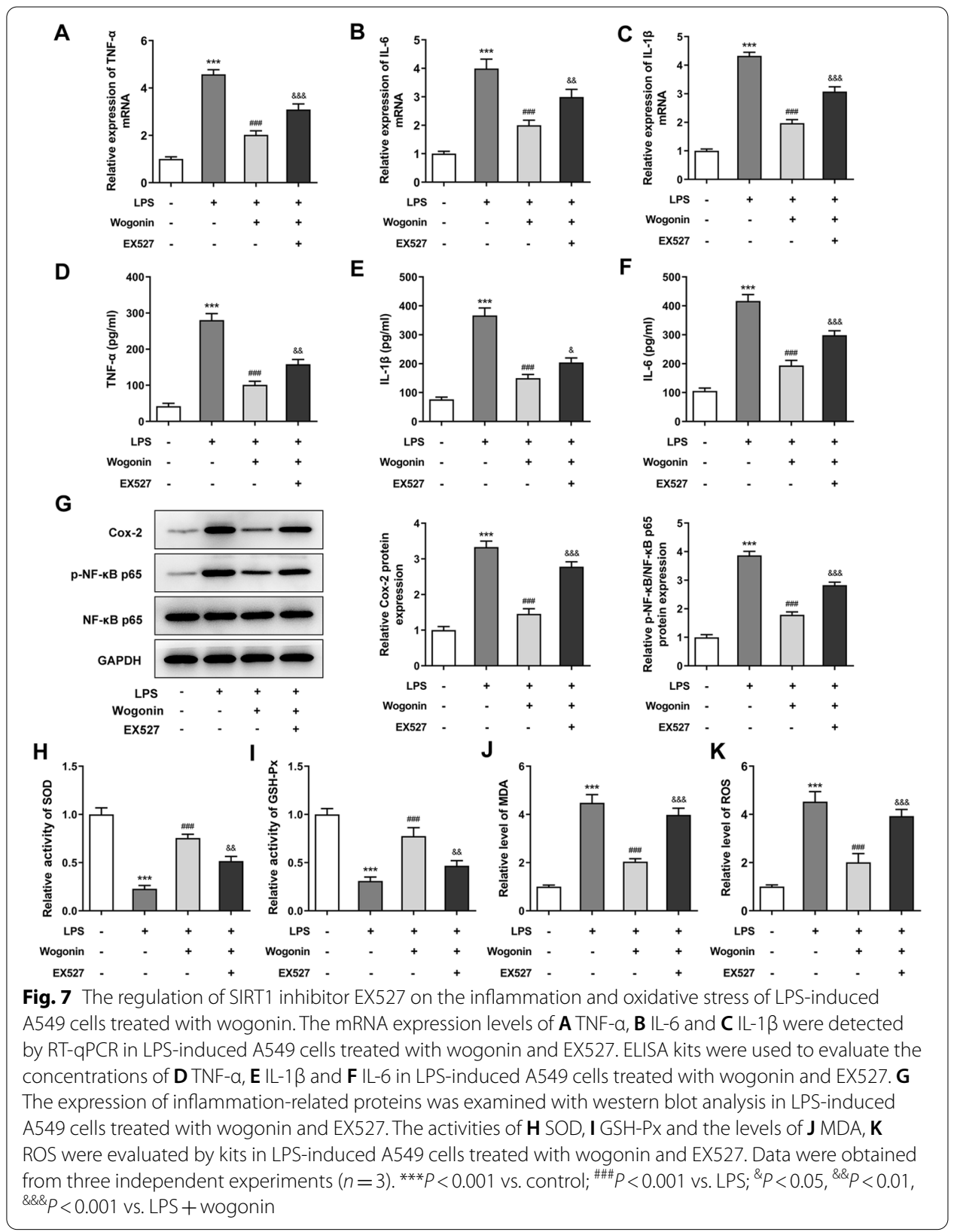

stress and inflammation are two major hallmarks in the pathogenesis of ALI, and thus we detected the changes in the oxidative stress indicators and inflammatory cytokines upon wogonin treatment [18]. Intriguingly, inflammation and oxidative stress were both alleviated by wogonin exposure in LPS-treated A549 cell model, as evidenced by the blockade of proinflammatory factor release and the enhanced production of SOD and GSH-Px, as well as decreased production of MDA after wogonin treatment.

HMGB1 emerges as a highly conserved protein that was first identified to modulate sepsis in a murine model [19]. Targeting HMGB1 was considered as a potential therapeutic option for the treatment of sepsis owing to the fact that patients who manifested 
as prolonged inflammatory responses frequently displayed continued high HMGB1 levels [10]. Recent reports and reviews have paid much attention to the therapeutic effects of HMGB1 on the inflammatory diseases, not limited to sepsis. For instance, it was previously demonstrated that the inhibition of HMGB1 by ulinastatin ameliorated the LPSinduced ALI injury in mice [10, 20]. Following being released from activated monocytes/ macrophages, HMGB1 served as a proinflammatory factor via various stimulants [20]. Inflammatory signals like LPS could prompt the acetylation-related translocation of HMGB1 from the nucleus to the cytoplasm [21]. Here, the finding that the translocation of HMGB1 from the nucleus to the cytoplasm upon LPS stimulation was counteracted by wogonin treatment indirectly elucidated that the inflammation of A549 cells triggered by LPS was impeded potently by wogonin.

SIRT1 has been suggested to play a significant role in various biological processes [22]. Depending on its deacetylation targets, alterations in the activity of SIRT1 was expectedly concerned with oxidative stress and inflammation [23]. HMGB1 was recently recognized as a deacetylation target of SIRT1 [20]. Substantial evidence indicated the SIRT1-modulated deacetylation of HMGB1 alleviated inflammation, restored renal function, and prolonged the survival time of mice with sepsis-related acute kidney injury [15]. In an experimental traumatic brain injury study, Omega-3 polyunsaturated fatty acid alleviated the inflammation by modulating microglia polarization through SIRT1modulated deacetylation of the HMGB1/NF- $\mathrm{kB}$ pathway [24]. Further, oleanolic acid prevented rat model from subarachnoid hemorrhage by SIRT1-modulated HMGB1 deacetylation [25]. Inhibition of NLRP3/NF-kB by Aloin via activation of SIRT1 attenuated the LPS-challenged ALI in mice [26]. By regulating SIRT1/HMGB1/NF-kB signaling, kaempferol improved lung injury induced by ischemia-reperfusion via anti-inflammation and anti-oxidative stress [27]. As an important molecule in the downstream of HMGB1, NF- $\mathrm{kB}$ is a major signaling pathway involved in the regulation of inflammatory mediators by activating proinflammatory factor genes and releasing a large number of inflammatory factors, including TNF- $\alpha$, IL- 6 and TNF-a $[28,29]$. In addition, NF-kB can also participate in the oxidative stress process via regulating the production of ROS and affecting the levels of SOD, MDA and GSH-Px [30]. In the present study, the decreased $\mathrm{p}-\mathrm{NF}-\mathrm{kB}$ p65 expression was observed after wogonin addition, which was restored by SIRT1 inhibitor, EX527. Additionally, we found that deacetylated HMGB1 by wogonin was accompanied by activated SIRT1 expression, which led to our speculation that wogonin might exert protective effects on LPS-induced inflammation of A549 cells by SIRT1-mediated HMGB1 deacetylation. The use of EX527 in the following experiments further demonstrated that inhibition of SIRT1 reversed the protective effects of wogonin on the inflammation of LPS-induced A549 cells.

\section{Conclusion}

In conclusion, we demonstrated that wogonin alleviated the inflammation, oxidative stress and apoptosis of the LPS-induced ALI cell model. This mechanism of action appeared to be owing to the regulation of SIRT1-mediated HMGB1 deacetylation triggered by wogonin. These findings might identify a novel mechanism by which wogonin exerts protective effects on ALI and provide an experimental basis for the application of wogonin to the clinical treatment of ALI. 


\section{Materials and methods}

\section{Cell culture and drugs}

Human lung epithelial cells (A549) were purchased from the Cell Bank of Shanghai Institute of Biochemistry and Cell Biology at the Chinese Academy of Sciences (Shanghai, China) and cultured in Dulbecco's Modified Eagle Medium (DMEM; Gibco, Paisley, UK) containing 10\% heat-inactivated fetal bovine serum (Gibco, Paisley, UK), 100 $\mathrm{U} / \mathrm{ml}$ penicillin, and $100 \mathrm{~g} / \mathrm{ml}$ streptomycin at $37{ }^{\circ} \mathrm{C}$ with $5 \% \mathrm{CO}_{2}$. The cells were collected after $10 \mu \mathrm{g} / \mathrm{mL}$ LPS (Sigma Chemical Co., St Louis, MO) stimulation for $24 \mathrm{~h}$. Wogonin (purity >99\%), isolated from Scutellaria baicalensis Georgi, was bought from Biotic Chemical (Taipei, Taiwan). It was dissolved in dimethylsulfoxide (DMSO), stored at $-20{ }^{\circ} \mathrm{C}$ and diluted by DMEM. SIRT1 inhibitor EX527 (10 $\mu \mathrm{M}$; MedChemExpress, Shanghai, China) was used to treat cells for $24 \mathrm{~h}$ before administration according to the previous study [31].

\section{Cell Counting Kit-8 (CCK-8) assay}

After the stimulation of LPS for $24 \mathrm{~h}$ and various concentrations of wogonin exposure for another $4 \mathrm{~h}$, cells were seeded into a 96-well plate and cell viability assay was performed by adding $10 \mu \mathrm{L}$ of CCK-8 reagent (Dojindo, Kumamoto, Japan) into each well at $37{ }^{\circ} \mathrm{C}$ for $4 \mathrm{~h}$. The absorbance at $450 \mathrm{~nm}$ was monitored using a microplate reader (BioRad Laboratories, Inc.).

\section{Lactate dehydrogenase $(\mathrm{LDH})$ release assay}

The cell supernatant treated with wogonin was seeded in 96-well culture plates and incubated for $24 \mathrm{~h}$ at $37{ }^{\circ} \mathrm{C}$ with $5 \% \mathrm{CO}_{2}$ before $100 \mu \mathrm{L}$ of the Cytotoxicity Detection Kit LDH solution (Roche Diagnostics, France) was added to each well. 15 min later, cell supernatant was diluted, with optical density value monitored at $450 \mathrm{~nm}$.

\section{Terminal-deoxynucleotidyl transferase-mediated nick end labeling (TUNEL) staining}

TUNEL assay was carried out to assess the apoptosis of LPS-induced A549 cells with or without wogonin treatment by Colorimetric TUNEL Apoptosis Assay Kit (Beyotime, Shanghai, China) based on the operation guidelines. Following phosphate buffer saline (PBS) washing for twice and fixation by $4 \%$ paraformaldehyde for $0.5 \mathrm{~h}, 0.3 \%$ hydrogen peroxide in PBS was used to incubate cells for another $20 \mathrm{~min}$ at room temperature. Cells were then treated by diaminobenzene (DAB) for $10 \mathrm{~min}$ and counterstained by hematoxylin (Solarbio, Beijing, China) for $30 \mathrm{~s}$. The apoptotic-positive cells were observed by a fluorescence microscope (Olympus Corporation) and the apoptotic rate was qualified by Image-J software (NIH, Bethesda, MD, USA).

\section{Acridine orange/ethidium bromide dual staining}

Acridine orange/ethidium bromide (AO/EB) double fluorescence assays were performed to evaluate cell apoptosis alteration. In brief, A549 cells were grown on glass coverslips in 24-well plates. The cells were pre-incubated for $24 \mathrm{~h}$ with serum-free medium, and then incubated with LPS and various concentrations of wogonin at $37^{\circ} \mathrm{C}$. The cells were washed with PBS and supplied with $5 \mu \mathrm{L}$ AO/EB mixed solution $(100 \mu \mathrm{g} / \mathrm{ml}$ of AO and $100 \mu \mathrm{g} / \mathrm{ml}$ of EB mixed in PBS, Aladdin, China) within $3 \mathrm{~min}$. The cells were observed 
and taken a photograph at $510 \mathrm{~nm}$ excitation wavelength under a fluorescence microscope (Olympus Corporation).

\section{Western blot analysis}

Proteins were extracted from A549 cells using RIPA lysis buffer (Beyotime, Shanghai, China) and the concentrations of proteins were measured by a bicinchoninic acid (BCA) Protein Assay Kit (Thermo Fisher Scientific, USA). Following that, 10\% sodium dodecyl sulfate-polyacrylamide gel electrophoresis (SDS-PAGE) electrophoresis was performed to separate $40 \mu \mathrm{g}$ proteins and the proteins were then transferred to polyvinylidene difluoride (PVDF) membranes. These membranes were then blocked by nonfat milk and then incubated at $4{ }^{\circ} \mathrm{C}$ overnight with primary antibodies. Afterwards, HRP-conjugated secondary antibody was used to incubate the membranes for $1.5 \mathrm{~h}$ at room temperature. Finally, bands were visualized by an enhanced chemiluminescence (ECL) kit (Amersham Biosciences, Buckinghamshire, UK), while band intensity was monitored using Image-J software (NIH, Bethesda, MD, USA). GAPDH was considered as an internal reference.

\section{Reverse transcription-quantitative PCR (RT-qPCR)}

A549 cells were lysed in $1 \mathrm{ml} \mathrm{TRIzol}{ }^{\circledR}$ reagent (Invitrogen, Carlsbad, CA, USA) for the extraction of total RNA. After the collection of total RNA, reverse transcription was conducted for complementary DNA (cDNA) synthesis by PrimeScript RT reagent kit (Takara Bio, Inc.). The conditions were as follows: $50{ }^{\circ} \mathrm{C}$ for $15 \mathrm{~min}, 85^{\circ} \mathrm{C}$ for $5 \mathrm{~s}$ and preservation at $4{ }^{\circ} \mathrm{C}$. SYBR Premix Ex Taq TM (TaKaRa, Japan) was used to set the PCR reaction conditions and reaction system based on the suggestions of the manufacturer. An ABI 7500 instrument (AB-4351107; Applied Biosystems; Thermo Fisher Scientific, Inc.) was used for qPCR. The following thermocycling conditions were used: initial denaturation at $95{ }^{\circ} \mathrm{C}$ for $10 \mathrm{~min}$; followed by 40 cycles of denaturation at $95{ }^{\circ} \mathrm{C}$ for $15 \mathrm{~s}$ and annealing at $60{ }^{\circ} \mathrm{C}$ for $1 \mathrm{~min}$; and a final extension of $10 \mathrm{~min}$ at $72{ }^{\circ} \mathrm{C}$. GAPDH was accepted as the normalization control of relative gene expression. Calculation of relative gene expression was conducted by $2^{-\Delta \Delta \mathrm{Ct}}$ method.

\section{Enzyme-linked immunosorbent assay (ELISA)}

The cellular levels of interleukin-6 (IL-6), IL-1 $\beta$ and tumor necrosis factor- $\alpha$ (TNF- $\alpha$ ) in the cell supernatant were measured using IL-6 ELISA Kit, IL-1 $\beta$ ELISA Kit and TNF- $\alpha$ ELISA Kit based on the suggestions of the manufacturer (Shanghai XiTang Biotechnology, Shanghai, China).

\section{Detection of oxidative stress}

For the detection of oxidative stress, the activities of oxidative stress markers including malondialdehyde (MDA), reactive oxygen species (ROS), glutathione peroxidase (GSH-Px) and superoxide dismutase (SOD) were detected following the manufacturer's instructions (Nanjing Jiancheng Biotechnology Institute, China).

\section{Statistical analysis}

All data were shown as mean \pm standard deviation (SD) of at least three independent experiments. For comparisons among multiple groups, one-way analysis of variance 
(ANOVA) with Tukey's post hoc test was carried out, while those between two groups were conducted by Student's $t$-test. $P<0.05$ was deemed as statistically significant.

\section{Abbreviations}

ALI: Acute lung injury; LPS: Lipopolysaccharide; HMGB1: High-mobility group box 1 protein; PPARy: Peroxisome proliferator-activated receptor gamma; MAPK: Mitogen Activated Protein Kinase; JNK: C-Jun NH(2)-terminal kinase; CLP: Cecal ligation and puncture; DMEM: Dulbecco's modified Eagle medium; DMSO: Dimethylsulfoxide; CCK-8: Cell counting kit-8; LDH: Lactate dehydrogenase; TUNEL: Terminal-deoxynucleotidyl transferase-mediated nick end labeling; PBS: Phosphate buffer saline; DAB: Diaminobenzene; AO/EB: Acridine orange/ethidium bromide; BCA: Bicinchoninic acid; SDS-PAGE: Sodium dodecyl sulfate-polyacrylamide gel electrophoresis; PVDF: Polyvinylidene difluoride; ECL: Enhanced chemiluminescence; cDNA: Complementary DNA; ELISA: Enzyme-linked immunosorbent assay; IL: Interleukin; TNF-a: Tumor necrosis factor-a; MDA: Malondialdehyde; GSH-Px: Glutathione peroxidase; SOD: Superoxide dismutase; SD: Standard deviation; ANOVA: Analysis of variance; Cox-2: Cyclooxygenase-2; Nrf2: Nuclear factor erythroid 2-related factor 2; ROS: Reactive oxygen species.

\section{Acknowledgements}

None.

\section{Authors' contributions}

JG: project development, data analysis and collection, manuscript writing. HY: project development, data collection. YZ: data analysis, manuscript editing. YL: project development, manuscript editing. All authors have read and approved the final manuscript.

\section{Funding}

None.

Availability of data and materials

The experimental data will be available on the request.

\section{Declarations}

Ethics approval and consent to participate

Not applicable.

\section{Consent for publication}

All authors have read the manuscript and agreed to submit it.

\section{Competing interests}

The authors declare they have no competing interests.

\section{Author details}

${ }^{1}$ Department of Pulmonary and Critical Care Medicine, Wenzhou Hospital of Integrated Traditional Chinese and Western Medicine, Wenzhou 325000, Zhejiang Province, China. ${ }^{2}$ Department of Respiratory and Critical Care Medicine, The Second People's Hospital of Nantong, 298 Xinhua Road, Chongchuan District, Nantong 226002, Jiangsu, China.

Received: 26 June 2021 Accepted: 29 November 2021

Published online: 14 December 2021

\section{References}

1. Mokra D, Kosutova P. Biomarkers in acute lung injury. Respir Physiol Neurobiol. 2015;209:52-8.

2. Hayes M, Curley G, Ansari B, Laffey JG. Clinical review: Stem cell therapies for acute lung injury/acute respiratory distress syndrome-hope or hype? Crit Care. 2012;16(2):205.

3. Angus DC, Clermont G, Linde-Zwirble WT, Musthafa AA, Dremsizov TT, Lidicker J, et al. Healthcare costs and longterm outcomes after acute respiratory distress syndrome: A phase III trial of inhaled nitric oxide. Crit Care Med. 2006;34(12):2883-90.

4. Hu JT, Lai J, Zhou W, Chen XF, Zhang C, Pan YP, et al. Hypothermia alleviated LPS-induced acute lung injury in rat models through TLR2/MyD88 pathway. Exp Lung Res. 2018;44(8-9):397-404.

5. Chi YS, Lim H, Park H, Kim HP. Effects of wogonin, a plant flavone from Scutellaria radix, on skin inflammation: in vivo regulation of inflammation-associated gene expression. Biochem Pharmacol. 2003;66(7):1271-8.

6. Wei CY, Sun HL, Yang ML, Yang CP, Chen LY, Li YC, et al. Protective effect of wogonin on endotoxin-induced acute lung injury via reduction of p38 MAPK and JNK phosphorylation. Environ Toxicol. 2017;32(2):397-403.

7. Yeh YC, Yang CP, Lee SS, Horng CT, Chen HY, Cho TH, et al. Acute lung injury induced by lipopolysaccharide is inhibited by wogonin in mice via reduction of Akt phosphorylation and RhoA activation. J Pharm Pharmacol. 2016;68(2):257-63. 
8. Yao J, Pan D, Zhao Y, Zhao L, Sun J, Wang Y, et al. Wogonin prevents lipopolysaccharide-induced acute lung injury and inflammation in mice via peroxisome proliferator-activated receptor gamma-mediated attenuation of the nuclear factor-kappaB pathway. Immunology. 2014;143(2):241-57.

9. Kwak S, Ku SK, Han MS, Bae JS. Vascular barrier protective effects of baicalin, baicalein and wogonin in vitro and in vivo. Toxicol Appl Pharmacol. 2014;281(1):30-8.

10. Luo Y, Che W, Zhao M. Ulinastatin post-treatment attenuates lipopolysaccharide-induced acute lung injury in rats and human alveolar epithelial cells. Int J Mol Med. 2017;39(2):297-306.

11. Li X, Mo J, Li J, Chen Y. IncRNA CASC2 inhibits lipopolysaccharide induced acute lung injury via miR27b/TAB2 axis. Mol Med Rep. 2020;22(6):5181-90.

12. Zeng $M$, Sang $W$, Chen $S$, Chen $R$, Zhang $H$, Xue F, et al. 4-PBA inhibits LPS-induced inflammation through regulating ER stress and autophagy in acute lung injury models. Toxicol Lett. 2017;271:26-37.

13. Huang $T$, Zhang $Y$, Wang $C$, Gao J. Propofol reduces acute lung injury by up-regulating gamma-aminobutyric acid type a receptors. Exp Mol Pathol. 2019;110: 104295.

14. Yang T, Liu H, Zhao B, Xia Z, Zhang Y, Zhang D, et al. Wogonin enhances intracellular adiponectin levels and suppresses adiponectin secretion in 3T3-L1 adipocytes. Endocr J. 2017;64(1):15-26.

15. Wei S, Gao Y, Dai X, Fu W, Cai S, Fang H, et al. SIRT1-mediated HMGB1 deacetylation suppresses sepsis-associated acute kidney injury. Am J Physiol Renal Physiol. 2019;316(1):F20-31.

16. Yao J, Zhao L, Zhao Q, Zhao Y, Sun Y, Zhang Y, et al. NF-kB and Nrf2 signaling pathways contribute to wogoninmediated inhibition of inflammation-associated colorectal carcinogenesis. Cell Death Dis. 2014;5(6): e1283.

17. Khan NM, Haseeb A, Ansari MY, Devarapalli P, Haynie S, Haqqi TM. Wogonin, a plant derived small molecule, exerts potent anti-inflammatory and chondroprotective effects through the activation of ROS/ERK/Nrf2 signaling pathways in human Osteoarthritis chondrocytes. Free Radic Biol Med. 2017;106:288-301.

18. Lang JD, MCArdle PJ, O'Reilly PJ, Matalon S. Oxidant-antioxidant balance in acute lung injury. Chest. 2002;122(6 Suppl):314S-S320.

19. Wang H, Ward MF, Sama AE. Targeting HMGB1 in the treatment of sepsis. Expert Opin Ther Targets. 2014;18(3):257-68.

20. Andersson U, Wang H, Palmblad K, Aveberger AC, Bloom O, Erlandsson-Harris H, et al. High mobility group 1 protein (HMG-1) stimulates proinflammatory cytokine synthesis in human monocytes. J Exp Med. 2000;192(4):565-70.

21. Bonaldi T, Talamo F, Scaffidi P, Ferrera D, Porto A, Bachi A, et al. Monocytic cells hyperacetylate chromatin protein HMGB1 to redirect it towards secretion. EMBO J. 2003;22(20):5551-60.

22. Yuk JM, Kim TS, Kim SY, Lee HM, Han J, Dufour CR, et al. Orphan nuclear receptor ERRa controls macrophage metabolic signaling and A20 expression to negatively regulate TLR-induced inflammation. Immunity. 2015;43(1):80-91.

23. Xu S, Gao Y, Zhang Q, Wei S, Chen Z, Dai X, et al. SIRT1/3 activation by resveratrol attenuates acute kidney injury in a septic rat model. Oxid Med Cell Longev. 2016;2016:7296092.

24. Chen X, Chen C, Fan S, Wu S, Yang F, Fang Z, et al. Omega-3 polyunsaturated fatty acid attenuates the inflammatory response by modulating microglia polarization through SIRT1-mediated deacetylation of the HMGB1/NF-kappaB pathway following experimental traumatic brain injury. J Neuroinflammation. 2018;15(1):116.

25. Han Y, Tong Z, Wang C, Li X, Liang G. Oleanolic acid exerts neuroprotective effects in subarachnoid hemorrhage rats through SIRT1-mediated HMGB1 deacetylation. Eur J Pharmacol. 2021;893: 173811.

26. Lei J, Shen Y, Xv G, Di Z, Li Y, Li G. Aloin suppresses lipopolysaccharide-induced acute lung injury by inhibiting NLRP3/NF-kappaB via activation of SIRT1 in mice. Immunopharmacol Immunotoxicol. 2020;42(4):306-13.

27. Yang C, Yang W, He Z, He H, Yang X, Lu Y, et al. Kaempferol improves lung ischemia-reperfusion injury via antiinflammation and antioxidative stress regulated by SIRT1/HMGB1/NF-kappaB axis. Front Pharmacol. 2019;10:1635.

28. Lawrence T. The nuclear factor NF-kappaB pathway in inflammation. Cold Spring Harb Perspect Biol. 2009;1(6): a001651.

29. Wang L, Ma H, Xue Y, Shi H, Ma T, Cui X. Berberine inhibits the ischemia-reperfusion injury induced inflammatory response and apoptosis of myocardial cells through the phosphoinositide 3-kinase/RAC-alpha serine/threonineprotein kinase and nuclear factor-kappaB signaling pathways. Exp Ther Med. 2018;15(2):1225-32.

30. Kleinschnitz C, Grund H, Wingler K, Armitage ME, Jones E, Mittal M, et al. Post-stroke inhibition of induced NADPH oxidase type 4 prevents oxidative stress and neurodegeneration. PLoS Biol. 2010. https://doi.org/10.1371/journal. pbio. 1000479

31. Li H, Hao Y, Yang LL, Wang XY, Li XY, Bhandari S, et al. MCTR1 alleviates lipopolysaccharide-induced acute lung injury by protecting lung endothelial glycocalyx. J Cell Physiol. 2020;235(10):7283-94.

\section{Publisher's Note}

Springer Nature remains neutral with regard to jurisdictional claims in published maps and institutional affiliations. 